\title{
Commentaries
}

\section{NOS inhibitors in colitis: a suitable case for treatment?}

Nitric oxide (NO) is now well characterised as a physiologically important molecule, acting most notably as an intra- and intercellular messenger within the cardiovascular and nervous systems. ${ }^{1}$ To fulfil these functions NO is produced in a tightly controlled manner by calcium dependent, constitutively expressed nitric oxide synthases (cNOS) present within endothelial and neural cells. Under pathological conditions, however, NO acts very differently from a benign signalling molecule. In these circumstances NO is produced in large, apparently tissue damaging amounts by inducible, calcium unregulated nitric oxide synthases (iNOS). For example, in inflammatory bowel disease the presence of high local concentrations of pro-inflammatory cytokines, such as interleukin 8 , is associated with the induction of iNOS and so the continuous local generation of $\mathrm{NO}^{2}{ }^{3}$ possibly in very great amounts. ${ }^{4}$ Notably, this difference in NO production between cNOS and iNOS enzymes is not due, as is often remarked, to the inducible isoform of NO synthase being capable of producing more NO than the constitutive forms. Indeed, cNOS and iNOS enzymes function with very similar kinetics, co-factor requirements and substrate affinities. The important characteristic of iNOS enzymes is that they are not regulated by intracellular calcium. So, once iNOS is expressed within a cell it immediately sets to work forming NO.

The characterisation of cNOS and iNOS permitted a neat theory to be put forward to explain the conflicting roles of NO. cNOS enzymes made NO in low concentrations as a signalling molecule, whereas iNOS enzymes made NO in high, cell damaging, concentrations. However, there was a problem with this theory. NO by itself is not especially reactive. So, how does the production of NO in ungoverned amounts contribute to the tissue damage seen in many inflammatory states? The answer to this question was provided by Beckman and colleagues ${ }^{5}$ who showed that the reaction of NO with superoxide anion was not a simple inactivation process ${ }^{6}$ but rather an important activation step, resulting in the formation of peroxynitrite $\left(\mathrm{ONOO}^{-}\right)$. Unlike NO, peroxynitrite is a reactive species, giving rise to further damaging molecules, most notably the extremely destructive hydroxyl anion $\left(\mathrm{OH}^{-}\right)$. The formation of reactive peroxynitrite by this combination between NO and superoxide anion explains why-for example, neutrophils are required to produce both species if they are to be effective killers of invading cells. ${ }^{5}$ In inflamed tissue we should also expect peroxynitrite to be formed, as iNOS is present (see earlier) along with considerable populations of invading cells, many of which produce superoxide anion. As long as the NO and superoxide anion are produced in close proximity we must conclude that peroxynitrite is formed, and importantly that this could be central to the inflammatory damage seen. Indeed, instillation of the highly reactive and short-lived peroxynitrite directly into the rat colon unsurprisingly causes notable local damage and inflammation. ${ }^{7}$ In this issue (see page 180), Kimura and colleagues, supporting the work of Singer et $a l,{ }^{8}$ provide further evidence that NO produced by iNOS within the colonic mucosa of active ulcerative colitis is directly associated with the local production of peroxynitrite. In essence, these authors have found iNOS to be present throughout the colonic mucosa in amounts that are proportional to the degree of mucosal inflammation. This does not show that peroxynitrite is also formed, and unfortunately because of its highly reactive nature there is no way to measure peroxynitrite concentrations directly within living tissues. However, peroxynitrite reacts with proteins leaving behind tell-tale nitrotyrosine residue "footprints". These "footprints" can be detected by selective antibodies, which Kimura et al have used to show that, as for iNOS, there is a clear correlation between nitrotyrosine groups and tissue inflammation.

Clearly, our most important question must be whether or not the observations of Kimura et al and others will lead to the use of NOS inhibitors as new therapies for inflammatory bowel disease. Unfortunately, our state of knowledge suggests that the answer to this question is no. Currently, there are few genuinely selective iNOS inhibitors and no reports of their use in animal models of these disease states. Non-selective cNOS and iNOS inhibitors have been tested and sometimes found to be therapeutically effective in animal models. ${ }^{9}$ However, these nonselective agents also inhibit cNOS very well and can exacerbate intestinal damage ${ }^{10}$ by both reducing local blood flow and promoting neutrophil adhesion to the blood vessel wall. ${ }^{11}$ Although it is hoped these side effects could be avoided by the use of selective iNOS inhibitors, it must be borne in mind that cNOS isoforms could also be up-regulated in inflamed tissues, making the selective targeting of a disease specific NOS isoform impossible. ${ }^{12}$ Also supporting our cautious conclusion are studies demonstrating that in a disease model closer to the human, rhesus monkeys displaying spontaneous chronic colonic inflammation, administration of NO synthase inhibitors reduces neither histological inflammatory scores nor diarrhoeal symptoms. ${ }^{13}$

One final point of interest arising from Kimura et al's study is, as remarked by the authors, that many of the subjects were taking steroids without any noticeable effect on iNOS expression or activity, or peroxynitrite formation. Steroids dramatically down-regulate iNOS expression in numerous cell and animal models. Does this reflect different dosing levels of steroids in animal models and human disease, different pathways of iNOS regulation in acute and chronic inflammatory states, or differences between the human and animal models? The data presented here do not permit us to draw a conclusion. However, as we investigate the actions of iNOS inhibitors in human disease states, it is clearly vital to understand why such a powerful inhibitor of iNOS expression was without effect.

T D WARNER

$S$ A MCCARTNEY

Vascular Inflammation,

The William Harvey Research Institute,

St Bartholomew's and the Royal London

School of Medicine and Dentistry,

Charterhouse Square,

London EC1M 6BQ, UK 


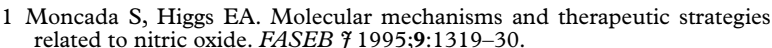

2 Boughton-Smith NK, Evans SM, Hawkey CJ, et al. Nitric oxide synthase activity in ulcerative colitis and Crohn's disease. Lancet 1993;342:338-40. 3 McLaughlan JM, Seth R, Vautier G, et al. Interleukin-8 and inducible nitric oxide synthase mRNA levels in inflammatory bowel disease at first presentation. F Pathol 1997;181:87-92.

4 Lundberg JO, Hellstrom PM, Lundberg JM, et al. Greatly increased luminal nitric oxide in ulcerative colitis. Lancet 1994;344:1673-4.

5 Beckman JS, Koppenol WH. Nitric oxide, superoxide, and peroxynitrite: the good, the bad, and the ugly. Am f Physiol 1996;271:C1424-37.

6 Gryglewski RJ, Palmer RMJ, Moncada S. Superoxide anion is involved in the breakdown of endothelium-derived relaxing factor. Nature 1986;320:454-6.

7 Rachmilewitz D, Stamler JS, Karmeli F, et al. Peroxynitrite-induced rat colitis - a new model of colonic inflammation. Gastroenterology 1993;105: 1681-8.

8 Singer TI, Kawka DW, Scott S, et al. Expression of inducible nitric oxide synthase and nitrotyrosine in colonic epithelium in inflammatory bowel disease. Gastroenterology 1996;111:871-85.

9 Hogaboam CM, Jacobson K, Collins SM, et al. The selective beneficial effects of nitric oxide inhibition in experimental colitis. Am f Physiol 1995; 268:G673-84.

10 Dobosz M, Mionskowska L, Dobrowolski S, et al. Is nitric oxide and heparin treatment justified in inflammatory bowel disease? An experimental study. Scand 7 Clin Lab Invest 1996;56:657-63.

11 Lopez-Belmonte J, Whittle BJ. Aminoguanidine-provoked leukocyte adherence to rat mesenteric venules: roles of constitutive nitric oxide synthase inhibition. Br f Pharmacol 1995;116:2710-14.

12 Iwashita E, Miyahara T, Hino K, et al. High nitric oxide synthase activity in endothelial cells in ulcerative colitis. F Gastroenterol 1995;30:551-4.

13 Ribbons KA, Currie MG, Connor JR, et al. The effect of inhibitors of inducible nitric oxide synthase on chronic colitis in rhesus monkey. F Pharmacol Exp Ther 1997;280:1008-15.

\section{Ambulatory manometry in dyspepsia: walking a thin line}

Fifteen years ago it was believed that motility studies would become to functional symptoms what endoscopy was (and still is) to ulcers, gallstones and cancer. Today, the ratio of endoscopic examinations to motility studies in most district general hospitals remains hundreds to one. There is no shortage of patients (about half the new referrals to gastroenterology outpatients are for functional symptoms), just no tests with which to make a positive diagnosis. The study by Wilmer et al from Leuven in Belgium illustrates why the early expectations of motility studies have not been realised (see page 235).

The starting point for the study was the belief that disturbed upper gut motility is responsible for functional dyspeptic symptoms. Others have shown that dyspeptic patients have abnormal motility. ${ }^{1}$ This study hoped to go one step further and link symptoms with specific patterns of dysmotility by using prolonged ambulatory motility studies. The logical extension of finding such a link is that the test would be able to distinguish those with and without the disorder and become clinically useful.

The investigators studied patients with "severe" symptoms and used the latest recording technology with both visual and computerised analysis of recordings. They found a high frequency of both qualitative and quantitative abnormalities of antrojejunal motility in the patient group. However, correlation of symptomatic episodes (there were 19 episodes in eight of 14 patients studied) with abnormal motility occurred only $21 \%$ of the time. In other words, there was a lot of abnormal motor activity in the absence of symptoms, and $79 \%$ of the time, when there were symptoms, the tracings were normal. Either the study design was unable to show a causal association when one exists, or there is none.

Three possible design errors that may have lead to missing a causal association include insufficiently sensitive manometric or analytical techniques, examination of the wrong segment of gut, or inappropriate selection of patients. It is difficult to imagine how, with available technology, the technical aspects of the study could be improved and the investigators gave good reasons to choose the antrum and upper intestine. Furthermore, identification of abnormal motor patterns suggests that the techniques and segment of gut chosen were appropriate. The failure of the study to link abnormalities with symptoms was probably unaffected by these factors. This leaves us with the possibility that either selection of patients affected the outcome, or that motility abnormalities are largely unconnected to functional dyspepsia.
We are told the patients had "severe" symptoms and that some had lost weight. There is no mention of other factors, unrelated to symptoms, that may have influenced referral and eventual inclusion into the study, nor whether the patients' problems were solely physical. The focus on the severity of symptoms and the complete absence of other information tells us that the authors work under the presumption that symptoms are the most important driving force in consultation behaviour, and in referral for investigation and subsequently for secondary and tertiary opinions. This oversight ignores an emerging and increasingly respectable literature on somatisation ${ }^{2}$ and everyday experience ${ }^{3}$ of unhappy, confused, anxious, and sometimes abused people with "severe" symptoms. Even weight loss (six of 14 patients had weight loss between 8 and $16 \mathrm{~kg}$ ) occurs in the depressed or emotionally disturbed. Moreover, how do we know the patients did not have an eating disorder?

The response of functional gut symptoms to cognitivebehavioural therapy, ${ }^{4}$ hypnotherapy ${ }^{5}$ and psychotherapy ${ }^{6}$ lends weight to the belief that psychological factors play an important role in their genesis. Ignoring these factors at the point of selection into motility studies is asking for a confused result. Excluding patients with significant psychological disturbance may improve the correlation between symptoms and dysmotility. Alternatively, it is possible that dysmotility is the intermediate event between stress and symptoms and that exclusion of stressed patients will result in a convincing negative correlation. Disregarding behavioural and psychological factors is a common occurrence in high tech motility studies and so it is little wonder manometry is not getting very far in the clinical arena.

The best investigators of motility (of whom those from Leuven are typical) are very good at dealing with the technical challenges they face. The achievement of doing and analysing such studies must not be underestimated or undervalued. Therefore, it is a shame that so little effort (in this case none) goes into the behavioural, cognitive and psychological factors that are associated with functional symptoms. Ignoring these devalues the studies, leads to inconclusive conclusions and leaves motility studies out in the cold, perhaps for another 15 years. Can we persuade the famous physiologists from Leuven (and their brethren elsewhere) to join forces on an equal footing with psychologists who understand the nuances of consultation behaviour and the complex relationship of psychological distress to somatic symptoms? Only then can we hope to tease out those with a primary motility disorder and enable motility studies to come of age.

Consultant Gastroenterologist,

R VALORI

Gloucester Gastroenterology Group,

Gloucestershire Royal Hospital,

Great Western Road,

Gloucester GL1 3NN, UK 
1 Jebbink HJA, van Berge-Henegouwen GP, Akkermans LMA, et al. Small intestinal motor abnormalities in patients with functional dyspepsia demonstrated by ambulatory manometry. Gut 1996:38:694-700.

2 Anonymous. The psychological care of medical patients. Recognition of need and service provision. A report of the Royal College of Physicians and the Royal College of Psychiatrists. RCP Publications, April 1995.

3 Valori R. Lines written on reading another review article about the irritable bowel syndrome. Lancet 1993;341:36-7.
4 Klimes I, Mayou RA, Pearce MJ, et al. Psychological treatment for atypical non-cardiac chest pain: a controlled evaluation. Psychol Med 1990;20:60511 .

5 Whorwell PJ, Prior A, Faragher EB. Controlled trial of hypnotherapy in the treatment of severe refractory irritable bowel syndrome. Lancet 1984; ii: 1232-4.

6 Guthrie E, Creed F, Dawson D, et al. A controlled trial of psychological treatment for the irritable bowel syndrome. Gastroenterology 1991;100:450-7.
Since the first descriptions of the contaminated small bowel syndrome, controversy has raged about both its pathogenesis and the mechanism of the malabsorption which often accompanies it. ${ }^{1-4}$ One, still popular, theory is that in small bowel bacterial overgrowth the concentration of conjugated bile acids in the gut lumen is reduced, by bacterial deconjugation, to levels less than those required for adequate micelle formation. The result is malabsorption of fat. However, there is as much controversy over the part played by bile acid deconjugation in the fat malabsorption of small bowel bacterial overgrowth as there is about whether malabsorption per se is an inevitable consequence of colonisation of the small bowel by colonic bacteria. Clearly, the presentation of this syndrome varies widely from patient to patient and a full investigation of the relation between events in the intestinal lumen and symptoms would therefore be very useful when considering treatment. However, there are many unresolved practical problems in this field, not least of which are the difficulties of quantitating fat malabsorption and assessing the bacterial flora of the small bowel.

The best test of fat malabsorption is generally considered to be a faecal fat measurement made on a standardised fat intake and stool collection over at least five days. Unfortunately, neither patients nor laboratory staff appreciate this ideal. Numerous alternatives have been proposed, the most successful of which is, perhaps, the ${ }^{14} \mathrm{C}$-triolein breath test. However, although this test's sensitivity and specificity are high, in practice the relation between fat digestion and fat malabsorption is far from linear. ${ }^{5}$ Consequently, the ${ }^{14} \mathrm{C}$-triolein test is of doubtful value in situations other than moderate to severe pancreatic insufficiency.

The classic way to diagnose small bowel bacterial overgrowth is to culture aspirates of the luminal contents of the small bowel. Although generally accepted as the gold standard, as many as three aspirates (near, mid and distal small bowel) may be required for diagnostic certainty. This need, together with that for facilities capable of detecting fragile anaerobes, often prohibits such an approach. A frequently used alternative is the bile acid breath test. ${ }^{6}$ The problems of interpreting the results of this test if it is applied with a protocol different from that described originally, are legendary. In the context of small bowel bacterial overgrowth it is insensitive and non-specific.

Fortunately, the bacteria of the large bowel are usually kept in their place, and by at least two mechanisms: gut propulsion and gastric acid secretion. The notion that diminished gastric acid secretion may be associated with intestinal bacterial overgrowth is not new. However, most studies have focused on the stomach and duodenum and apart from work by Shindo and colleagues, ${ }^{78}$ little attention has been given to possible changes in the jejunal flora. Shindo et al found that jejunal aspirates from patients and volunteers receiving cimetidine were overgrown with bacteria. The in vitro ability of these aspirates to deconjugate gycocholic acid mirrored positive in vivo bile acid breath tests, the results of which were significantly reduced towards normal by administration of tetracycline. Last year, in a prospective, randomised trial, Thorens et al found, perhaps not surprisingly, that treatment with omeprazole was more likely to produce gastric and duodenal overgrowth than was that with cimetidine. ${ }^{9}$ Thorens et al, who assessed bacterial overgrowth by aspiration and culture, did not aspirate the jejunal lumen but they did conclude that neither treatment induced malabsorption . . . as indicated by no changes in serum vitamin B12, beta carotene and albumin!

In this issue (see page 266) Shindo et al report that 12 of 21 patients (the oldest was 71 ) treated with omeprazole developed jejunal bacterial overgrowth. Most of the bacterial species present in jejunal fluid, aspirated from a site 30 $\mathrm{cm}$ past the ligament of Treitz, after just two weeks of omeprazole $(20 \mathrm{mg} /$ day), were capable of deconjugating the bile acids present in ox bile. Omeprazole treatment was also associated with significant increases in the exhaled ${ }^{14} \mathrm{CO}_{2}$ during the bile acid breath test, which were eliminated by co-administration of oral tetracycline for one week. The authors emphasise that they did not determine whether the stomach or the jejunum was the site of the breath test deconjugation (they were using a modified breath test which allowed no firm conclusions to be drawn as to the site of deconjugation). Nevertheless, omeprazole treatment was associated with fat malabsorption as judged by the ${ }^{14} \mathrm{C}$-triolein breath test, and tetracycline apparently restored fat absorption to normal.

Although many questions are raised by this study (such as what are the relations among flora composition, flora migration, $\mathrm{pH}$ and propulsion, and what is the effect of the ileal brake mechanism on the bile acid breath test profile?), the authors have attempted to delineate, for perhaps the first time, the link between bacterial overgrowth, bile acid deconjugation and fat malabsorption in a group of patients receiving omeprazole. A clinical colleague tells me that although malabsorption is not listed as a side effect of omeprazole treatment, diarrhoea is. This raises the interesting question of what clinical importance to lend to Shindo et al's observations.

G M MURPHY

Division of Medicine,

Gastroenterology Unit,

5th Floor, Thomas Guy House,

Guys Hospital,

London SE1 9RT, UK

1 Donaldson RM. Studies on the pathogenesis of steatorrhea in the blind loop syndrome. F Clin Invest 1965;44:1815-25.

2 Gorbach SI, Tabaqchali S. Bacteria, bile and the small bowel. Gut 1969;10: 963-72.

3 Gracey MJ.The contaminated small bowel syndrome: pathogenesis, diagnosis, and treatment. Am f Clin Nutr 1979;32:234-43.

4 Isaacs PET, Kim YS. The contaminated small bowel syndrome. Am f Med Isaacs PET, Kim Y
1979;67:1049-57. 
5 Lembcke B, Braden B, Caspary WF. Exocrine pancreatic insufficiency: accuracy and clinical value of the uniformly labelled ${ }^{13} \mathrm{C}-\mathrm{Hiolein}$ breath accuracy and clinical value

6 From $\mathrm{H}, \mathrm{Hofmann}$ AF. Breath test for altered bile acid metabolism. Lancet From H, Hofm.

7 Shindo K, Fukumura $M$. Effect of $\mathrm{H}_{2}$-receptor antagonists on bile acid metabolism. F Invest Med 1995;43:170-7.
8 Shindo K, Yamazaki R, Koide K, et al. Alteration of bile acid metabolism by cimetidine in healthy humans. F Invest Med 1996;44:462-9.

9 Thorens J, Froehlich F, Schwizer W, et al. Bacterial overgrowth during treatment with omeprazole compared with cimetidine: a prospective randomised double blind study. Gut 1996;39:54-9.

\section{Coming clean on reuse of endoscopic equipment}

Over the past decade, endoscopic accessories have evolved from reusable to disposable in many parts of the world. The impetus for this has been multifactorial and includes concerns of cross-contamination between patients because of inability to assure proper cleaning and the inevitable equipment deterioration that occurs with reuse. ${ }^{1}$ A contrasting and more cynical view has been espoused, however, that marketing items as "one-time-use" precludes the need for manufacturers to undertake microbiological studies after reprocessing. Moreover, its implementation has been associated with a dramatic increase in the purchase of accessories such as needle injectors, sphincterotomes, and even biopsy forceps. ${ }^{2}$ Within the United States, several factors came together to reinforce this "use and dispose" mentality. On the one hand are the known personnel costs needed to store and reprocess an instrument. On the other are medicolegal concerns regarding both cross-contamination between patients and use of an accessory that failed to maintain optimal form and function. Couple the former with an insurance system that routinely reimbursed for the use and disposal of such devices and the fact that many such accessories were only available in a disposable version (for example, double- and triple-lumen sphincterotomes), it is little wonder that the endoscopic accessory market has increased 10-fold in the United States over the past decade.

Several problems have occurred with the shift to disposable accessories in the United States which have direct applicability to European endoscopists and to the report published in this issue (see page 304). On the one hand, the manufacture of disposable accessories utilises a disproportionate percentage of the Earth's increasingly limited resources. On the other, their disposal is regulated by law in most countries and whether they are burned, buried or, paradoxically, recycled, disposal of medical supplies and devices has raised important environmental concerns. Finally, there is the issue of cumulative cost. As insurance programs in the United States evolved into new payment policies that reimbursed at very discounted levels, and as the government Medicare and Medicaid programs reimbursed hospitals for a diagnosis (DRG), not upon the resources used during that hospital stay; institutions became increasingly aware that disposable accessories may not be reimbursed. In other words, an institution could charge whatever it likes but reimbursement was limited contractually at a pre-set fee level.

Our group has evaluated many aspects of the reusabledisposable accessory question. ${ }^{3-7}$ For instance, we have suggested that the expense associated with "one-time-use" accessories for therapeutic endoscopic retrograde cholangiopancreatography are such that this procedure may ultimately lose money for the hospital contingent upon other expenses expended (personnel and medication costs, admit-recovery charges...). ${ }^{3}$ Our data have been confirmed recently by other groups. ${ }^{8}$ We have also looked at items that are available in both a disposable and reusable form and have documented purchase and reprocessing costs in our unit prospectively, demonstrating that biopsy forceps became cost effective after seven uses at the time our study was done. ${ }^{4}$ Finally, to cut our costs further, we have evaluated the reuse of items marketed as "one-time-use" when there were no comparable accessories marketed for multiple use. This was not done lightly, and for double-channel sphincterotomes included testing of electrical integrity and contaminating 10 sphincterotomes with an atypical mycobacteria followed by manual processing and sterilisation with ethylene oxide gas on 10 occasions. ${ }^{6}$ These data were, in turn, presented to an institutional Reuse Committee comprised of three surgeons, a gastroenterologist, head of the microbiology laboratory, the chief operating room nurse, an infectious disease nurse, head of central supply, and the hospital attorney. ${ }^{5}$ Finally, following approval for reuse, a one-year prospective trial was undertaken to assure that there was not an increase in untoward infectious events related to the reuse of these devices. ${ }^{7}$

This has been an expensive and time consuming process and has recently been foreshortened by a number of entrepreneurial reprocessing companies in the United States. Such companies incorporate testing facilities to assure that "one-time-use" items can be safely reprocessed without loss of form and function. ${ }^{9}$ Not limited to endoscopic accessories, reprocessed devices run the gamut from laparoscopic trochars to pneumatic stockings for the postoperative patient to cardiology devices such as balloons or electro-ablation catheters. The companies assure sterilisation and adequate functioning and carry a cumulative liability coverage up to 10 million dollars if device dysfunction is associated with patient injury. However, they charge $50 \%$ of the original purchase price to reprocess an accessory.

Does the experience in the United States shed any light on how to solve the dilemma of reusing a single-use endoscopic accessory in the United Kingdom following publication of MDA DB 9501 and implementation of the European Medical Devices Directive? The simple answer is to purchase only reusable accessories. This is easier said than done, however, although it seems that some of the manufacturers have heard the laments from colleagues on both sides of the ocean. As such, at least two manufacturers will market reusable double-channel sphinterotomes over the next few months. Whether individual hospital or government regulations in Britain will allow the labour intensive and variably expensive approaches for reuse outlined earlier (Reuse Committee and reprocessing companies) is uncertain. Perhaps the best approach to reuse, however, is to define accurately the cost structure of your endoscopy unit. As such, modern units are likely to stock a variety of accessories based upon initial purchase price, documented ability to reprocess and reprocessing costs, and the number of uses that can be expected for a reusable or "reposable" device. Like all solutions, what works and is cost effective in one setting may prove of marginal or no benefit in another. One thing is certain, however: medical costs cannot continue to climb indefinitely. Cost con- 
straints will then either push competing manufacturers into reducing prices or limit the number of disposable accessories which are ultimately purchased.

R A KOZAREK

Section Head,

Section of Gastroenterology,

Virginia Mason Medical Center,

1100 Ninth Avenue, PO Box 900 (C3-GAS),

Seattle, WA 98111, USA

1 Sullivan P. Speakers offer vastly different views on disposable health care products. Can Med Assoc F 1990;143:1342-55.

2 Bruning LM. Disposable v. reusables in OR practice. Part II. Weighing costs, risks, and wastes. Nurs Manage 1992;23:721-P.
3 Kim-Deobald J, Kozarek RA, Ball TJ, et al. Prospective evaluation of costs of disposable accessories in diagnostic and therapeutic ERCP. Gastrointest Endosc 1993;39:763-5.

4 Kozarek RA, Raltz SL, Merriam LD, et al. Disposable versus reusable biopsy forceps: a prospective evaluation of costs. Gastrointest Endosc 1996;43:1013.

5 Kozarek RA. Reuse of disposable equipment: one medical center's approach to the problem [abstract]. Gastrointest Endosc 1995;41:323.

6 Kozarek RA, Sumida SE, Raltz SL, et al. In vitro evaluation of wire integrity and ability to reprocess single-use sphincterotomes. Gastrointest Endosc 1996;45:117-21.

7 Kozarek RA, Raltz SL, Ball TJ, et al. One-year prospective study reusing disposable sphincterotomes for diagnostic and therapeutic ERCP. Gastrointest Endosc (in press).

8 Walker RS, Vanagunas AD, Williams P, et al. Therapeutic ERCP: a cost-prohibitive procedure? Gastrointest Endosc 1997;46:143-6.

9 Anonymous. Reuse of single-use medical devices. Third-party reprocessing company audit tool. Plymouth, PA: Emergency Case Research Incorporated, 1997:76. 